\title{
İkramiye Vadisi Florası (Sapanca) Sakarya
}

\author{
Melike TURNA ${ }^{1}$, Mehmet SAĞIROĞLU ${ }^{1 *}$ \\ ${ }^{1}$ Sakarya Üniversitesi, Fen Edebiyat Fakültesi, Biyoloji Bölümü, 54087, SAKARYA
}

\section{Öz}

Bu çalı̧̧mada İkramiye vadisi (Sapanca/Sakarya) ve çevresinin floristik özellikleri araştırılmıştır. Yapılan arazi çalışmaları sonucunda, araştırma alanında 79 familyaya ait 257 cins, 434 tür ve tür altı seviyede takson belirlenmiștir. Bu taksonlardan 5'i Pteridophyta bölümüne, 429’u Spermatophyta bölümüne aittir. Spermatophyta bölümüne ait olan taksonlardan 5'i Gymnospermae, 424'ü Angiospermae alt bölümündedir. Florayı oluşturan taksonların fitocoğrafik bölgelere göre dağılımı şöyledir; 110 takson Avrupa-Sibirya, 51 takson Akdeniz, 14 takson İran-Turan fitocoğrafik bölgesine aittir, fitaocoğrafik bölgesi bilinmeyen takson sayısı 259'tır. Bölgede bulunan taksonların hayat formlarına göre dağılımları ise şöyledir; 52'si Fanerofit, 36's1 Kamefit, 172'si Hemikriptofit, 56'sı Kriptofit, 121'i Terofit ve 1 tanesi Vasküler parazittir. Araştırma alanındaki endemik takson sayısı 11 olup endemizm oranı \%2,53’tür. Araştırma alanında tespit edilen 29 takson A3 karesi için yeni kayıttır.

Anahtar Kelimeler: Flora, İkramiye Vadisi, Sapanca, Sakarya, Türkiye.

\section{Flora of Ikramiye Valley (Sapanca) Sakarya}

\begin{abstract}
In this study, İkramiye Valley's and its surrounding's floristical features were investigated. As a result of field studies, 434 taxa belong to 257 genera and 79 families, were identified in the research area. Of these 5 taxa are reserved in the Pteridophyta section and 429 are in the Spermatophyta section. 5 taxa belong to the Spermatophyta section are included in the Gymnospermae and 424 taxa are in the Angiospermae section. The distribution of the taxa, according to phytogeographic region, are as follows; 110 taxa belong to Euro-Siberian; 51 taxa Mediterranean; 14 taxa Iran-Turanian phytogeographic region and 259 taxa are not known which phytogeographic region they belong. According to the life forms; 52 taxa belong to Phanerophytes, 36 Chamaephytes, 172 Hemicryptophytes, 56 Cryptophytes, 121 Therophytes, 1 Vascular parasites. The number of endemic taxon is 11 and the endemism rate is \% 2.53. 29 taxa are new record for the A3 square in the research area.
\end{abstract}

Key Words: Flora, Ikramiye Valley, Sapanca, Sakarya, Turkey. 


\section{Giriş}

Sakarya ili Sakarya nehri havzası ve bu havzaya bağlı alanların dışında ovalar, tepeler ve dağlardan oluşur. Bu alanların bitki örtüsü dağlık ve tepelik alanlarda doğal olarak saf veya karışık ormanlar ile örtülüdür. Bu ormanlar yüksek kesimlerde iğne yapraklı aşağı kesimlerde meşelerin ağırlıkta olduğu karışık topluluklar halindedir. Yükseltinin deniz seviyesine yaklaştığı alanlarda Akdeniz iklimi benzer bir iklimin etkisi ile maki-pseudomaki topluluklarının sık görüldüğ̈̈ bir bitki örtüsü vardır.

Sakarya ilindeki toprakların yaklaşık \% 45'i ormanlar, \%40 ekili-dikili alanlar, \%10'u çayır ve meralar, \% 5 kadarı tarıma elverişsiz alanlardan ibarettir. İl genelinde Sakarya nehri ve havzasındaki alanların tamamına yakınında ekilebilir-dikilebilir tarım yapılmaktadır. Bu alanlar genelde sebze ve meyve yetiştiriciliği için kullanılmaktadır. İkramiye vadisi Sapanca ilçesi sınırları içinde olup, Sapanca’ ya uzaklığı 13 km, Sakarya iline uzaklığı 30 km'dir. Güneyinde Pamukova ve Geyve ilçeleri, kuzeyinde merkez ilçe olan Adapazarı, doğusunda Karapürçek ve Akyazı ilçeleri, batısında ise İzmit ili bulunmaktadır. İkramiye vadisi Sapanca'nın güneyinde yer almaktadır. Vadi içinde başta İkramiye olmak üzere Fevziye, İlmiye, Memnuniye, Şükriye ve Nailiye köyleri bulunmaktadır(http://www.googlearth).

Çalışma bölgesi yamaçlar, vadiler, dere kenarları, tarım alanları, yol kenarları ve ormanlık alanlardan oluşmaktadır. Araştırma alanının en alçak yeri Akçay köyü, en yüksek yeri İkramiye köyünün tepe kısmıdır. Araştırma alanı Avrupa-Sibirya Fitocoğrafik bölgesinde ve A3 Grid karesinde yer almaktadır.

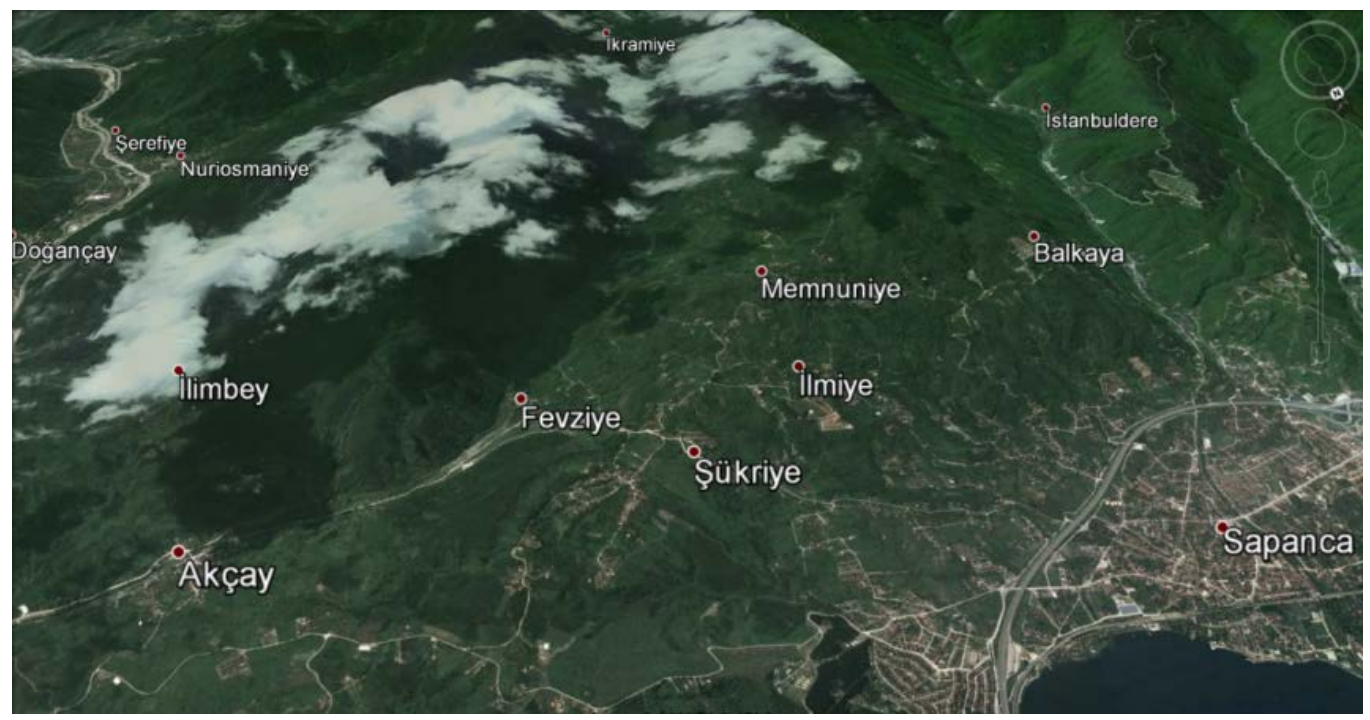

Şekil 1.Çalışma alanı coğrafi konumu görüntü.

Çalışma alanının toprak gruplarını kahverengi orman toprakları, kireçsiz kahverengi orman toprakları ve alüvyal topraklar oluşturmaktadır. Araştırma alanı Akdeniz iklimi ile Yarı Akdeniz iklimi arasında bir geçiş bölgesinde yer almaktadır (Akman, 2011).

Çalıma alanı; yamaçlar, vadiler, dere yatakları, yol kenarları, çalılıklar, geni ve ine yapraklı karışık ormanlardan meydana gelmektedir. Alan doğal olarak Avrupa-Sibirya fitocoğrafik bölgesinde yer aldığı ve düzenli yağış rejimine sahip olduğu için step vejetasyonu görülmez. Ancak, antropojenik etkiler sonucu bazı tepe ve yamaçların bitki örtüsünün tahrip edilmesi ile step tarzı bir çayır vejetasyonu ortaya çıkmaktadır. Çayır vejetasyonu daha çok yamaçlarda görülmektedir. Bunun yanında doğal vejetasyon iğne yapraklı ve yaprak döken ağaç topluluklarının karışık olarak meydana getirdiği orman vejetasyonudur. Orman vejetasyonunun tahrip edilmesi ile açılan alanlarda genelde findik tarımı yapılmaktadır. Sakarya ilinde farklı kültürlerin oluşturduğu bir nüfus bulunmaktadır. Bu farklı kültürlerde bitkilerin tıbbı kullanımını ortaya koyan çalışmalar tarafımızdan daha önce yapılmıştır(Sağıroğlu vd., 2012, Sağıroğlu vd., 2017).

\section{Materyal ve Metot}

Araştırma alanında 2011-2013 yılları arasında belirli periyotlarla toplam 16 kez arazi çalışması yapılmıştır. Bu süre içinde 608 bitki örneği toplanmıştır. Örnekler mümkün olduğunca çiçekli ve meyveli olarak toplanmıştır. 
Toplanan örnekler kurallara uygun olarak preslenip kurutularak herbaryum materyali haline getirilmiştir. $\mathrm{Bu}$ örneklerin teşhisi sonucu tür ve türaltı seviyede 426 takson tespit edilmiştir.

Teşhisler sırasında Flora of Turkey and Aegean Islands Vol. 1-9 (Davis, 1965-1985), Flora of Turkey and Aegean Islands Vol. 10 (Davis, 1988), Flora of Turkey and Aegean Islands (suppl. 2) Vol. 11 (Güner vd., 2000), Türkiye Bitkileri Listesi kaynaklarından yararlanılmıştır.

Ayrıca kültür bitkilerinin ve ağaçların teşhisinde Türkiye'nin Ağaçları ve Çalıları (Namıkolu, 2010), Süs bitkileri ve Yer Örtücüleri (Acartürk, 2001) adlı kitaplardan, bitki adları otörlerinin kontrolü için Authors of Plant Names (Brummitt, 1999) kitabından ve IPNI (www.ipni.org, 25.11.2013) den, yararlanılmıştır.

Floristik liste hazırlanırken Türkiye florası esas alındı. Bitkilerin listesi verilirken önce familyası yazıldı. Daha sonra cins, tür ve varsa tür altı taksonları otörleri ile birlikte verildi. Daha sonrada türün toplandığı il, yer veya mevkii adı, toplandığı yerin yüksekliği, tarihi, toplayıcı numarası, hayat formu, biliniyorsa hangi fitocoğrafik bölge elementi olduğu, geni yayılışlı ve endemik olup olmadığı sırasıyla verilmiştir. Çalışmada kullanılan kısaltmalar tablo 1'de verilmiştir.

Tablo 1. Kullanılan kısaltmalar.

\begin{tabular}{llll}
\hline D.Akd. & Doğu Akdeniz elementi & Te. & Terofit \\
Akd. & Akdeniz elementi & Vp. & Vasküler parazit \\
Avr.-Sib. & Avrupa-Sibirya elementi & Ök. & Öksin \\
İr.-Tur & İran-Turan elementi & End. & Endemik \\
Fa. & Fanerofit & CR & Çok tehlikede \\
Hkrp. & Hemikriptofit & LC & En az endişe verici \\
Krp. & Kriptofit & NT & Tehdit altına \\
& & & girebilir \\
Kam. & Kamefit & VU & Zarar görebilir \\
Gnş. Yay. & Geniş yayılışlı & & \\
\hline
\end{tabular}

\section{Bulgular}

\subsection{Bitki Toplanan İstasyonlar}

1. Akçay köyü, yol kenarı, 100 m. 2. Akçay köyü, Akçay okulu önü, yol kenarı, 100m.3. Akçay köyü, dere kenarı, 0-100 m. 4. Akçay-Memnuniye köy yolu, revan su fabrikasından sonra $1 . \mathrm{km}$, yol kenarı, 0-150m. 5. AkçayMemnuniye köy yolu, revan su fabrikasından sonra 1.km, taşlık alan, 100-150m. 6. Akçay-Memnuniye köy yolu, revan su fabrikasından sonra 2.km, açık düzlük alanlar, 100-150m.7. Fevziye köyü girişi, yol kenarı, 100-150m. 8. Fevziye köyü giriş̧i, açık alanlar, 100-200m.9. Fevziye köyü girişi, dere kenarı, 100-150m. 10. Fevziye köyü girişi, karışık orman açıklığı, 100-150m. 11. Fevziye köyü girişi, karışık orman açıklığı, 400m.12. Fevziye köyü orman içi, 400m.13. Murat köprüsü köyü girişi, nur mescidi karşısı, yol kenarı, 150-200m. 14. Murat köprüsü köyü girişi, nur mescidi önü, bahçe, $150-200 \mathrm{~m}$. 15. İlmiye köyü girişi, karışık orman açıklığ $1,200 \mathrm{~m}$. 16. İlmiye köyü, orman içi, 200m. 17. Memnuniye köyü, yol kenarı, 200-300m.18. Memnuniye köyü, orman içi, 200-300m.19. Memnuniye köyü, karışık orman açıklı̆̆ı, 250-300m. 20. Memnuniye köyü, dere kenarı, 100-200m. 21. Memnuniye köyü, yamaç, kumlu toprak, 200-300m.22. Memnuniye köyü, 3.km, orman içi, 200-300m.23. Memnuniye köyü, 4.km, orman içi, 200-300m.24. Memnuniye köyü, 4.km, su kenarı, 250-300m. 25. Memnuniye köyü, 5.km, orman içi, 200-300m.26. Memnuniye köyü, 6.km, yol kenarı, 200-300m. 27. Memnuniye köyü, 6.km, yamaç, 200-300m.28. Memnuniye köyü, 6.km, yamaç, 300-350m. 29. Memnuniye köyü, 6.km, orman içi, 300350m. 30. Memnuniye köyü, yol kenarı, su kenarı, 250-300m. 31. Memnuniye köyü çıkışı, açık alanlar, 250-300m. 32. Memnuniye köyü çıkışı, orman içi, 250-300m. 33. Memnuniye köyü çıkışı, açık alanlar, nemli toprak, 250$300 \mathrm{~m}$. 34. Memnuniye-İkramiye köy yolu, birinci köprüye gelmeden, yol kenarı, 200-300m.35. Memnuniyeİkramiye köy yolu, 4.km, orman içi, 200-300m. 36. Memnuniye-İkramiye köy yolu, $6 . \mathrm{km}$, yol kenarı, 200-300m. 37. Memnuniye-İkramiye köy yolu, $6 . \mathrm{km}$, yamaç, $200-300 \mathrm{~m} .38$. Memnuniye-İkramiye köy yolu, yol kenarı, 200300m. 39. İkramiye köyü girişi, yol kenarı, 250-300m. 40. İkramiye köyü, yol kenarı, 350-400m. 41. İkramiye köyü, yol kenarı, 450-600m. 42. İkramiye köyü, düzlük alan, yerleşim yeri, 500-550 m. 43. İkramiye köyü orman içi, 500-600m. 44. İkramiye köyü, vadi içi, yamaç, 500-600m. 45. İkramiye köyü, orman içi, dere kenarı, 350400m. 46. İkramiye köyü çıkışı, mezarlık önü, yol kenarı, 300-350m. 


\subsection{Bitki Listesi}

\section{PTERIDOPHYTA}

EQUISETACEAE

Equisetum giganteum L., 45, 13.04.2013, M.Turna 1401. Krp.

Equisetum arvense L., 17, 05.05.2012, M.Turna 1202. Krp.

Equisetum telmateia Ehrh.,43, 13.04.2013, M.Turna 1402. Krp.

HYPOLEPIDACEAE

Pteridium aquilinum (L.) Kuhn, 16, 31.07.2011, M.Turna 1079. Krp. Gnş. Yay..

POLYPODIACEAE

Polypodium vulgare L. var. vulgare, 43, 13.04.2013, M.Turna 1422. Hkrp.

SPERMATOPHYTA

\section{GYMNOSPERMAE}

\section{PINACEAE}

Abies nordmanniana (Steven) Spach subsp. equi-trojani (Asc. \& Sint. ex Boiss.) Coode \& Cullen, 20, 25.03.2012, M.Turna 1095. Fa. Ök. End.

Pinus sylvestris L. var. hamata Steven, 26, 25.03.2012, M.Turna 1102. Fa. Avr.-Sib.

CUPRESSACEAE

Cupressus sempervirens L., 13, 08.07.2013, M.Turna 1526. Fa. Kültür.

Juniperus oxycedrus L. subsp. oxycedrus, 14, 08.07.2013, M.Turna 1529. Fa. Gnş. Yay.

Thuja orientalis L., 36, 08.07.2013, M.Turna 1544. Fa. Kültür.

ANGIOSPERMAE

DICOTYLEDONAE

RANUNCULACEAE

Clematis vitalba L., 8, 03.06.2012, M.Turna 1190; 42, 22.07.2012, M.Turna 1306.Kam.

Clematis viticella L., 27.11.2011, M.Turna 1092. Kam.

Ranunculus constantinopolitanus (DC.) d ' Urv., 43, 13.04.2013, M.Turna 1441. Hkrp. Geniş yayıliş11.

Ranunculus marginatus d' Urv. , 8, 05.05.2012, M.Turna 1173. Te.

BERBERIDACEAE

Epimedium pubigerum (DC.) C.Morren \& Decne., 21, 05.05.2012. Krp. Ök.

BIGNONIACEAE

Campsis radicans (L.) Seem, 17, 08.07.2013, M.Turna 1500. Fa. Kültür.

PAPAVERACEAE

Chelidonium majus L., 18, 05.05.2012, M.Turna 1147. Hkrp. Avr.-Sib.

Papaver rhoeas L., 8, 03.06.2012, M.Turna 1185. Te. Gnş. Yay.

Papaver dubium L. subsp. dubium, 8, 31, 05.05.2012, M.Turna 1152.Te.

CRUCIFERAE

Brassica oleracea L., 42, 13.04.2013, M.Turna 1443. Hkrp. Kültür.

Diplotaxis viminea (L.) DC., 15, 21.03.2013, M.Turna 1394. Te.

Diplotaxis muralis (L.) DC., 8, 05.05.2012, M.Turna 1170. Te.

Raphanus raphanistrum L. subsp. raphanistrum, 32, 25.05.2013, M.Turna 1489. Te.

Calepina irregularis (Asso) Thellung, 8, 21.03.2013, M.Turna 1387. Te.

Lepidium draba L., 17, 05.05.2012, M.Turna 1119. Hkrp. Gnş. Yay.

Isatis tinctoria L. subsp. tomentella (Boiss.) Davis, 35, 19.06.2011, M.Turna 1036. Hkrp.

Isatis buschiana Schischk., 37, 08.07.2013, M.Turna 1531. Hkrp. Ir.-Tur.

Thlaspi jaubertii Hedge, 35, 19.06.2011, M.Turna 1029-1046. Te. End.

Teesdalia coronopifolia (Berg.)Thellung, 16, 25.03.2011, M.Turna 1111. Te. Akd.

Capsella bursa-pastoris (L.) Medik., 16, 25.03.2011, M.Turna 1109. Te. Kozmopolit.

Andrzeiowskia cardamine Rchb., 12, 01.06.2011, M.Sağıroğlu 3097. Te.

Draba verna L., 41, 13.04.2013, M.Turna 1421. Te.

Barbarea vulgaris R. Br. subsp. vulgaris, 19,23, 05.05.2012, M.Turna 1148. Hkrp.

Cardamine bulbifera (L.) Crantz, 43, 13.04.2013, M.Turna 1436. Krp. Avr.-Sib.

Cardamine impatiens L. subsp. impatiens L., 43, 13.04.2013, M.Turna 1409. Te. Avr.-Sib.

Cardamine impatiens L. subsp. pectinata (Pall.ex DC.) Stoj. \& Stef. 43, 13.04.2013, M.Turna 1408.Te. Avr.Sib.

Cardamine hirsuta L., 12, 01.06.2011, M.Sağıroğlu 3078.Te. Kozmopolit.

Erysimum cuspidatum (Bieb.) DC., 31, 20.09.2012, M.Turna 1321. Hkrp. Gnş. Yay.

Sisymbrium altissimum L., 12, 01.06.2011, M.Sağıroğlu 3084. Hkrp.

Sisymbrium irio L., 8, 05.05.2012, M.Turna 1168. Te.

Sisymbrium loeselii L., 12, 01.06.2011, M.Sağıroğlu 3061.Te. Gnş. Yay. 
Arabidopsis thaliana (L.) Heynhold, 17, 43, 05.05.2012, M.Turna 1181Te.

Camelina sativa (L.) Crantz, 35, 19.06.2011, M.Turna 1047. Te.

\section{CISTACEAE}

Cistus creticus L., 8, 31.07.2011, M.Turna 1081. Kam. Akd.

Cistus salviifolius L., 12, 01.06.2011, M.Sağıroğlu 3094. Kam.

VIOLACEAE

Viola odorata L., 18, M.Turna 1403. Hkrp.

Viola suavis Bieb., 41, 25.03.3012, M.Turna 1094. Hkrp.

Viola reichenbachiana Jord. ex Bor., 31, 43, 05.05.2012, M.Turna 1144. Hkrp.

Viola gracilis Sibth. \& Sm., 43, 13.04.2013, M.Turna 1426. Hkrp.

POLYGALACEAE

Polygala supina Schreb. subsp. supina., 6, 25.05.2013, M.Turna 1490. Hkrp. Gnş. Yay.

Polygala pruinosa Boiss. subsp. pruinosa, 43, 13.04.2013, M.Turna 1418. Hkrp. Geniş yayılışl1.

Polygala vulgaris L., 35, 19.06.2011, M.Turna 1039. Hkrp. Avr.-Sib.

Polygala monspeliaca L., 17, 31, 03.06.2012, M.Turna 1199. Te. Akd.

CARYOPHYLLACEAE

Arenaria serpyllifolia L. subsp. serpyllifolia, 6, 25.05.2013, M.Turna 1482. Te.

Arenaria leptoclados (Reichb.) Guss., 17, 13.04.2013, M.Turna 1446. Te.

Stellaria media (L.) Vill. 16, 25.03.2011, M.Turna 1110. Te.

Stellaria pallida (Dumort.) Piré, 22, 21.03.2013, M.Turna 1395. Te.

Stellaria holostea L., 31, 05.05.2012, M.Turna 1138. Hkrp. Avr.-Sib.

Cerastium dichotomum L. subsp. dichotomum, 15, 03.06.2012, M.Turna 1215. Te.

Cerastium glomeratum Thuill., 17, 13.04.2013, M.Turna 1445. Te. Kozmopolit.

Moenchia mantica (L.) Bartl, 16, 05.05.2012, M.Turna 1124; 15, 03.06.2012, M.Turna 1231. Te.

Dianthus calocephalus Boiss., 17, 18.07.2011, M.Turna 1059. Hkrp.

Dianthus giganteus d’ Urv., 23, 19.06.2011, M.Turna 1048. Hkrp. Avr.-Sib.

Silene italica (L.) Pers. subsp. italica, 11, 01.06.2011, M. Sağıroğlu3046. Hkrp.

Silene vulgaris (Moench) Garcke var. vulgaris, 40, 03.06.2012, M.Turna 1224. Hkrp.

Silene compacta Fisch. ex Hornem., 12, 19.06.2011, M.Turna 1019; 17, 18.07.2011, M.Turna 1058; 16,

31.07.2011, M.Turna 1082; 8, 20.06.2012, M.Turna 1261. Te.

Silene armeria L., 31, 31.07.2011, M.Turna 1084. Hkrp. Avr.-Sib.

Silene gallica L., 31, 25.03.2012, M.Turna 1093. Hkrp. Kozmopolit.

\section{ILLECEBRACEAE}

Scleranthus perennis L. subsp. marginatus (Guss.) Arc., 42, 13.04.2013, M.Turna 1432. Hkrp.

POLYGONACEAE

Polygonum Iapathifolium L., 8, 17.10.2012, M.Turna 1364. Te.

Rumex scutatus L., 12, 01.06.2011, M.Sağıroğlu 3089. Hkrp.

CHENOPODIACEAE

Chenopodium album L. subsp. album var. album, 8, 17.10.2012, M.Turna 1369. Te.

PHYTOLACCACEAE

Phytolacca americana L., 8, 20.06.2012, M.Turna 1271. Kam.

GUTTIFERAE

Hypericum calycinum L., 17, 31, 03.06.2012, M.Turna 1203. Kam. Ök.

Hypericum androsaemum L., 32, 22.07.2012, M.Turna 1300. Kam.

Hypericum lydium Boiss., 8, 23, 19.06.2011, M.Turna 1031. Hkrp. Ir.-Tur.

Hypericum montbretii Spach, 23, 19.06.2011, M.Turna 1042. Hkrp.

Hypericum cerastioides (Spach) N.Robson, 11, 01.06.2011, M.Sağıroğlu 3048. Hkrp.

Hypericum origanifolium Willd. var. origanifolium, 27, 08.07.2013, M.Turna 1514. Hkrp.

Hypericum tetrapterum Fries var. tetrapterum, 11, 01.06.2011, M.Sağıroğlu 3050. Krp.

Hypericum perforatum L. subsp. veronense (Schrank) H.Linb., 32, 08.07.2013, M.Turna 1523. Hkrp.

MALVACEAE

Malva sylvestris L.,2, 08.07.2013, M.Turna 1505. Hkrp.

Malva neglecta Wallr., 42, 13.04.2013, M.Turna 1543. Te.

Alcea biennis Winterl, 17, 08.07.2013, M.Turna 1511. Hkrp.

TILIACEAE

Tilia tomentosa Moench, 17, 03.06.2012, M.Turna 1220. Fa. Avr.-Sib.

LINACEAE

Linum trigynum L., 15, 31.07.2011, M.Turna 1072. Te. Akd.

Linum tenuifolium L., 26, 08.07.2013, M.Turna 1535. Hkrp. Akd. 
Linum bienne Miller,1, 6, 25.05.2013, M.Turna 1455; 1, 08.07.2013, M.Turna 1504. Hkrp. GERANIACEAE

Geranium purpureum Vill., 8, 05.05.2012, M.Turna 1157. Te.

Geranium rotundifolium L., 39, 05.05.2012, M.Turna 1143a. Te.

Geranium pusillum Burm. fil., 31, 05.05.2012, M.Turna 1143b. Te.

Geranium columbinum L., 8, 03.06.2012, M.Turna 1252. Te.

Geranium dissectum L., 8, 05.05.2012, M.Turna 1167. Te.

Geranium tuberosum L., 12, 01.06.2011, M.Sağıroğlu 3084. Krp.

Geranium pyreniacum Burm. fil., 8, 03.06.2012, M.Turna 1251. Krp.

Erodium ciconium (L.) L 'Hérit., 17, 13.04.2013, M.Turna 1444. Te.

Erodium cicutarium (L.) L 'Hérit. subsp. cicutarium, 8, 20.06.2012, M.Turna 1270. Te.

Erodium acaule (L.) Becherer \& Thell., 8, 21.03.2013, M.Turna 1386. Te. Akd.

OXALIDACEAE

Oxalis corniculata L., 8, 17.10.2012, M.Turna 1368. Te. Kozmopolit.

ACERACEAE

Acer platanoides L., 17, 05.05.2012, M.Turna 1117. Fa. Avr.-Sib.

STAPHYLEACEAE

Staphylea pinnata L., 31, 05.05.2012, M.Turna 1136-1140. Kam.

VITACEAE

Vitis vinifera L., 46, 13.04.2013, M.Turna 1542. Fa. Kültür.

RHAMNACEAE

Paliurus spina-christi P. Mill., 8, 20.06.2012, M.Turna 1265. Kam.

ANACARDIACEAE

Rhus coriaria L., 4, 25.05.2013, M.Turna 1471-1476. Kam.

\section{CELASTRACEAE}

Euonymus verrucosus Scop., 31, 22.07.2012, M.Turna 1299. Kam.

Euonymus latifolius (L.) Miller subsp. latifolius, 31, 05.05.2012, M.Turna 1135. Kam. Avr.-Sib.

LEGUMINOSAE (FABACEAE)

Cytisus hirsutus L., 12, M.Turna 1450. Kam.

Cytisus austriacus L., 23, 56, 19.06.2011, M.Turna 1035. Kam. Avr.-Sib.

Teline monspessulana (L.) C.Koch, 17, 18.07.2011, M.Turna 1060. Fa. Akd.

Genista tinctoria L., 17, 03.06.2012, M.Turna 1221.Fa. Avr.-Sib.

Genista sessilifolia DC., 42, 13.04.2013, M.Turna 1429. Kam. Ir.-Tur.

Robinia pseudoacacia L., 16, 05.05.2011, M.Turna 1128. Fa.

Galega officinalis L., 8, 03.06.2012, M.Turna 1195; 3, 08.07.2013, M.Turna 1519.

Hkrp. Avr.-Sib.

Bituminaria bituminosa (L.) C.H.Stirt. 4, M.Turna 1478. Hkrp. Akd.

Vicia cracca L. subsp. cracca, 43, 25.05.2013, M.Turna 1493. Hkrp. Avr.-Sib.

Vicia hirsuta (L.) S. F. Gray, 31, 03.06.2012, M.Turna 1229. Te.

Vicia lathyroides L., 43, 13.04.2013, M.Turna 1417. Te.

Vicia sativa L. subsp. nigra (L.) Ehrh. var. nigra, 16, 05.05.2012, M.Turna 1123. Te.

Lathyrus venetus (Miller) Wohlf., 12, 13.04.2013, M.Turna 1440. Kam. Avr.-Sib.

Lathyrus palustris L. subsp. palustris, 12, 19.06.2011, M.Turna 1012. Hkrp. Avr.-Sib.

Lathyrus laxiflorus (Desf.) O. Kuntze subsp. laxiflorus, 12, 19.06.2011, M.Turna 1008. Hkrp.

Lathyrus undulatus Boiss., 12, 01.06.2011, M.Sağıroğlu 3083. Hkrp. Ök. End.

Lathyrus nissolia L., 43, 25.05.2013, M.Turna 1492. Te.

Lathyrus aphaca L. var. biflorus Post, 43, 13.04.2013, M.Turna 1451. Te.

Ononis spinosa L. subsp. leiosperma (Boiss.) Širj., 6, 25.05.2013, M.Turna 1469. Hkrp. Gnş.Yay.

Trifolium repens $\mathrm{L}$. var. repens, 8, 05.05.2012, M.Turna 1156. Krp.

Trifolium nigrescens Viv. subsp. nigrescens, 8, 17.10.2012, M.Turna 1370. Te.

Trifolium campestre Schreb. subsp. campestre var. campestre, 6, 25.05.2013, M.Turna 1494. Te.

Trifolium fragiferum L. var. fragiferum, 8, 03.06.2012, M.Turna 1315. Krp.

Trifolium resupinatum L. var. resupinatum Rouy \& Fouc., 8, 05.05.2012, M.Turna 1155.Te.

Trifolium tomentosum L. var. tomentosum, 6, 25.05.2013, M.Turna 1495. Te.

Trifolium pratense L. var. pratense, 8, 10, 03.06.2012, M.Turna 1188.Hkrp. Gnş. Yay.

Trifolium pratense L. var. sativum Schreb., 23, 19.06.2011, M.Turna 1025. Hkrp.

Trifolium pallidum Waldst. \& Kit., 42, 13.04.2013, M.Turna 1425. Krp.

Trifolium ligusticum Balb. \& Lois. 8, 03.06.2012, M.Turna 1186. Te. Akd.

Trifolium cherleri L., 8, 03.06.2012, M.Turna 1187. Te. Akd.

Trifolium arvense L. var. arvense, 8, 20.06.2012, M.Turna 1257Te. Gnş. Yay. 
Trifolium angustifolium L, 23, 19.06.2011, M.Turna 1052. Te.

Trifolium constantinopolitanum Ser., 8, 05.05.2012, M.Turna 1160. Te. Gnş. Yay.

Melilotus albus Desr., 8, 22.07.2012, M.Turna 1284. Te. Geniş yayılışlı.

Medicago lupulina L., 8, 03.06.2012, M.Turna 1228. Hkrp. Geniş yayılışl1.

Medicago minima (L.) Bart. var. minima, 8, 05.05.2012, M.Turna 1159. Te. Gnş. Yay.

Dorycnium graecum (L.) Ser., 31, 03.06.2012, M.Turna 1209. Hkrp. Ök.

Dorycnium pentaphyllum Scop. subsp. herbaceum (Vill.) Rouy, 6, 25.05.2013, M.Turna 1491. Kam.

Lotus corniculatus L. var. corniculatus, 31, 20.09.2012, M.Turna 1333. Hkrp. Gnş. Yay.

Lotus corniculatus L. var. tenuifolius L., 8, 03.06.2012, M.Turna 1193. Hkrp. Gnş. Yay.

Hymenocarpos circinnatus (L.) Savi, 8, 05.05.2012, M.Turna 1171. Te. Akd.

Anthyllis hermanniae L., 31, 20.09.2012, M.Turna 1322. Hkrp. Akd.

Ornithopus compressus L., 12, 19.06.2012, M.Turna 1010. Te. Akd.

ROSACEAE

Laurocerasus officinalis M.Roem., 17, 05.05.2012, M.Turna 1121Fa.

Prunus spinosa L., 18, 21.03.2013, M.Turna 1398. Fa. Avr.-Sib.

Prunus x domestica L., 13, 08.07.2013, M.Turna 1501. Fa. Kültür.

Prunus divaricata Ledeb. var. divaricata, 12, 19.06.2011, M.Turna 1015. Fa. Gnş. Yay.

Cerasus avium (L.) Moench, 16, 05.05.2012, M.Turna 1129. Fa.

Rubus caesius L., 16, 31.07.2011, M.Turna 1080. Kam. Gnş. Yay.

Rubus sanctus Schreber, 8, 20.06.2012, M.Turna 1267. Kam. Gnş. Yay.

Rubus canescens DC. var. canescens, 8, 03.06.2012, M.Turna 1250; 41, 25.05.2013, M.Turna 1454. Kam.

Geniş yayılışlı.

Rubus hirtus Waldst. \& Kit., 23, 19.06.2011, M.Turna 1043. Kam. Avr.-Sib.

Potentilla reptans L., 23, 19.06.2011, M.Turna 1034-1038. Gnş. Yay.

Potentilla micrantha Ramond ex DC., 28, 25.03.2012, M.Turna 1098. Hkrp.

Fragaria vesca L., 16, 05.05.2012, M.Turna 1179. Hkrp.

Sanguisorba minor Scop. subsp. muricata (Spach) Briq., 31, 17.10.2012, M.Turna 1379. Hkrp. Gnş. Yay.

Rosa canina L., 23, 19.06.2011, M.Turna 1024. Kam.

Mespilus germanica L., 43, 13.04.2013, M.Turna 1442. Fa. Ök.

Pyracantha coccinea M.Roem., 16, 25.03.2012, M.Turna 1113. Kam.

Crataegus microphylla K. Koch subsp. microphylla , 31, 22.07.2012, M.Turna 1320. Kam. Ök.

Cydonia oblonga Miller, 41, 13.04.2013, M.Turna 1420. Fa.

Malus sylvestris Miller subsp. orientalis (A. Uglitzkich) Browicz var. orientalis, 31, 250-300 m, 03.06.2012,

M.Turna 1205. Fa.

Pyrus communis L. subsp. communis, 43, 25.05.2013, M.Turna 1452. Fa.

LYTHRACEAE

Lythrum salicaria L., 9, 22.07.2012, M.Turna 1283.Hkrp. Avr.-Sib. Gnş. Yay.

ONAGRACEAE

Epilobium hirsutum L., 31, 22.07.2012, M.Turna 1303Krp.

Epilobium parviflorum Schreber, 8, 20.09.2012, M.Turna 1354. Krp.

Epilobium montanum L., 31, 20.09.2012, M.Turna 1336. Krp. Avr.-Sib.

DATISCACEAE

Datisca cannabina L., 17, 18.07.2011, M.Turna 1069. Kam.

CRASSULACEAE

Sedum pallidum M.Bieb., 31, 03.06.2012, M.Turna 1207. Hkrp.

UMBELLIFERAE

Eryngium creticum Lam., 8, 22.07.2012, M.Turna 1292. Hkrp. Akd.

Eryngium campestre L. var. virens Link, 8, 22.07.2012, M.Turna 1290. Hkrp.

Chaerophyllum byzantinum Boiss., 17, 18.07.2011, M.Turna 1068. Hkrp. Ök.

Sium sisarum L. var. Iancifolium (Bieb.) Thell., 31, 22.07.2012, M.Turna 1308. Krp.

Oenanthe silaifolia Bieb., 15, 03.06.2012, M.Turna 1216. Krp. Gnş. Yay.

Ammi visnaga (L.) Lam., 31, 17.10.2012, M.Turna 1373. Te. Ak.

Ferulago galbanifera (Miller) W. Koch, 27, 18.07.2011, M.Turna 1064. Hkrp.

Laserpitium hispidum Bieb., 17, 18.07.2011, M.Turna 1067. Hkrp. Avr.-Sib.

Torilis arvensis (Huds.) Link subsp. arvensis, 31, 31.07.2011, M.Turna 1085. Te.

Caucalis platycarpos L., 8, 22.07.2012, M.Turna 1282. Te.

Daucus carota L., 31, 22.07.2012, M.Turna 1310. Hkrp.

Daucus guttatus Sibth. \& Sm. , 15, 31.07.2011, M.Turna 1076.Hkrp.

ARALIACEAE

Hedera helix L f. helix, 22, 21.03.2013, M.Turna 1397. Kam. 
Hedera colchica (K.Koch) K.Koch, 32, 08.07.2013, M.Turna 1538. Kam. Ök. CORNACEAE

Cornus sanguinea L. subsp. australis (C. A. Meyer ) Jáv, 23, 19.06.2011, M.Turna 1028. Fa. Avr.-Sib. CAPRIFOLIACEAE

Sambucus ebulus L., 8, 20.06.2012, M.Turna 1256. Hkrp. Avr.-Sib.

Sambucus nigra L., 31, 22.07.2012, M.Turna 1307. Kam. Avr.-Sib.

RUBIACEAE

Sherardia arvensis L., 17, 13.04.2013, M.Turna 1447.Ter. Akd.

Crucianella angustifolia L., 4, 25.05.2013, M.Turna 1457. Te. Akd.

Asperula pestalozzae Boiss., 23, 19.06.2011, M.Turna 1033. Te. Ök. End

Asperula tenella Heuffel ex Degen, 17, 18.07.2011, M.Turna 1065. Hkrp.

Asperula taurina L. subsp. taurina, 31, 05.05.2012, M.Turna 1141. Hkrp.

Asperula involucrata Wahlenb., 32, 08.07.2013, M.Turna 1540. Hkrp. Ök.

Galium elongatum C.Presl, 12, M.Turna 1004. Te. Avr.-Sib.

Galium verum L. subsp. verum, 8, 20.06.2012, M.Turna 1268. Hkrp. Avr.-Sib.

Galium verum L. subsp. glabrescens Ehrend., 23,19.06.2011, M.Turna 1055. Hkrp. Ir-Tur.

Galium incanum Sm. subsp. elatius (Boiss.) Ehrend., 28, 08.07.2013, M.Turna 1532. Hkrp. Ir-Tur.

Galium aparine L., 32, 08.07.2013, M.Turna 1539. Krp.

Galium tenuissimum M.Bieb. f. trichophorum (Kar. \& Kir.) Ehrend. \& Schönb.-Tem. 31, 03.06.2012, M.Turna 1204.Te. Ir.-Tur.

Rubia peregrina L., 152, 19.06.2011, M.Turna 1013. Hkrp. Akd.

VALERIANACEAE

Valeriana alliariifolia Adams, 20, 25.05.2013, M.Turna 1462. Hkrp.

DIPSACACEA

Dipsacus laciniatus L., 32, 22.07.2012, M.Turna 1278. Hkrp.

Knautia integrifolia (L.) Bert. var. bidens (Sm.) Borbás, 41, 25.05.2013, M.Turna 1461.Te. Akd.

Scabiosa columbaria L. subsp. columbaria var. columbaria, 8, 20.06.2012, M.Turna 1253. Hkrp.

Scabiosa columbaria L. subsp. ochroleuca (L.) Čelak. var. ochroleuca (L.) Coulter,17, 18.07.2011, M.Turna

1062. Hkrp.

Scabiosa atropurpurea L., 8, 20.06.2012, M.Turna 1274. Hkrp.

COMPOSITAE

Xanthium papposa L. subsp. italicum, 8, 17.10.2012, M.Turna 1366. Te.

Telekia speciosa (Schreber) Baumg., 32, 22.07.2012, M.Turna 1311. Hkrp. Avr.-Sib.

Asteriscus spinosus (L.) Sch.Bip., 8, 03.06.2012, M.Turna 1191. Te. Akd.

Inula germanica L., 31, 20.09.2012, M.Turna 1340. Hkrp. Avr.-Sib.

Inula viscosa (L.) Aiton, 10, 17.10.2012, M.Turna 1365. Hkrp. Akd.

Pulicaria dysenterica (L.) Bernh., 8, M.Turna 1285. Krp.

Solidago virgaurea L. subsp. virgaurea, 31, 20.09.2012, M.Turna 1323Hkrp.

Erigeron daenensis Vierh., 8, 20.09.2013, M.Turna 1360. Krp. Ir.-Tur.

Conyza bonariensis (L.) Cronquist, 8, 20.09.2012, M. Turna 1361. Te.

Bellis perennis L., 16, 25.03.2012, M.Turna 1106. Hkrp. Avr.-Sib.

Doronicum orientale Hoffm., 12, 13.04.2013, M.Turna 1414. Krp.

Senecio aquaticus Hill subsp. erraticus (Bertol.) Matthews, 12, 01.06.2011, M.Sağıroğlu 3071.Te. Avr.-Sib.

Senecio vulgaris L., 8, 21.03.2013, M.Turna 1390. Te.

Senecio vernalis Waldst \& Kit., 8, 03.06.2012, M.Turna 1196. Te. Gnş. Yay.

Tussilago farfara L., 18, 25.03.2012, M.Turna 1104. Krp. Avr.-Sib.

Petasites hybridus (L.) Gaertner, 30, 25.03.2012, M.Turna 1115. Hkrp. Avr.-Sib.

Calendula officinalis L., 22, M.Turna 1396. Te. Kültür.

Eupatorium cannabinum L., 32, 22.07.2012, M.Turna 1309. Kam. Avr.-Sib.

Anthemis cretica L. subsp. pontica (Willd.) Grierson, 31, 20.09.2012, M.Turna 1341. Hkrp.

Anthemis cretica L. subsp. tenuiloba (DC.) Grierson, 23, 19.06.2011, M.Turna 1041. Hkrp.

Anthemis cotula L., 6, 08.07.2013, M.Turna 1479. Hkrp.

Anthemis tinctoria L. var. pallida DC., 12, 01.06.2011, M.Sağıroğlu 3069. Hkrp.

Tanacetum parthenium (L.) Schultz Bip., 32, 08.07.2013, M.Turna 1524. Hkrp.

Tanacetum vulgare L., 32, 08.07.2013, M.Turna 1521. Hkrp.

Cirsium ligulare Boiss., 8, 05.05.2012, M.Turna 1151. Hkrp.

Cirsium hypoleucum DC., 43, 25.05.2013, M.Turna 1498. Hkrp. Ök.

Cirsium creticum (Lam.) d 'Urv. subsp. creticum, 31, 03.06.2012, M.Turna 1213. Hkrp. Akd.

Cirsium arvense (L.) Scop, 26, 08.07.2013, M.Turna 1508. Hkrp.

Picnomon acarna (L.) Cass., 31, 22.07.2012, M.Turna 1313. Te. Akd. Gnş. Yay. 
Centaurea inermis Velen., 32, 22.07.2012, M.Turna 1318. Hkrp.

Centaurea virgata Lam. 27, 08.07.2013, M.Turna 1534. Kam.

Centaurea iberica Trev. ex. Sprengel, 8, 17.10.2012, M.Turna 1367. Te. Gnş. Yay.

Centaurea calcitrapa L. subsp. calcitrapa, 23, 19.06.2011, M.Turna 1040. Hkrp. Akd.

Carthamus lanatus L., 32, 22.07.2012, M.Turna 1317. Te. Gnş. Yay.

Xeranthemum cylindraceum Sm., 8, 20.06.2012, M.Turna 1254. Te.

Scolymus hispanicus L., 8, 20.06.2012, M.Turna 1276. Hkrp. Akd.

Cichorium intybus L., 15, 31.07.2011, M.Turna 1077. Hkrp. Gnş. Yay.

Leontodon hispidus L. subsp. hispidus, 8, 21.03.2013, M.Turna 1393. Hkrp.

Helminthotheca echioides (L.) Holub, 8, 20.09.2012, M.Turna 1358. Te.

Hedypnois rhagadioloides (L.)Hayek subsp. cretica, 8, 03.06.2012, M. Turna 1161. Te. Akd.

Sonchus asper (L.) Hill subsp. glaucescens (Jordon) Ball, 8, 05.05.2012, M.Turna 1169. Hkrp. Gnş. Yay.

Lactuca tuberosa Jacq, 34, 13.04.2013, M.Turna 1438. Hkrp.

Lactuca saligna L., 10, 22.07.2012, M.Turna 1289. Hkrp.

Lactuca muralis (L.) Gaertn., 8, 22.07.2012, M.Turna 1298. Hkrp. Avr.-Sib.

Lapsana communis L. subsp. intermedia (Bieb.) Hayek, 23, 19.06.2011, M.Turna 1056. Hkrp. Geniş yay1lışl1.

Taraxacum serotinum (Waldst. \& Kit. ) Poiret, 42, 13.04.2013, M.Turna 1428.

Crepis alpestris (Jacq.) Tausch, 31, 17.10.2012, M.Turna 1378. Hkrp. Avr.-Sib. Hkrp. Geniş yayılışl1.

CAMPANULACEAE

Campanula lyrata Lam. subsp. lyrata, 8, 03.06.2012, M.Turna 1194. Hkrp. Gnş. Yay. End.

Campanula rapunculoides L. subsp. rapunculoides, 12, 19.06.2011, M.Turna 1005. Hkrp. Avr.-Sib.

Campanula ajugifolia Schult, 23, 19.06.2011, M.Turna 1037. Hkrp. Ir.-Tur. End.

Campanula grandis Fisch. \& C.A.Mey. subsp. grandis, 23, 19.06.2011, M.Turna 1023. Hkrp. Ök. End.

Asyneuma rigidum (Willd. ) Grossh. subsp. rigidum, 27, 08.07.2013, M.Turna 1528.

Legousia pentagonia (L.) Thellung, 41, 25.05.2013, M.Turna 1460.Te. Akd. Hkrp. Ir.-Tur

ERICACEAE

Rhododendron ponticum L. subsp. ponticum, 17, 05.05.2012, M.Turna 1120. Fa. Ök.

Erica arborea L., 12, 01.06.2011, M.Sağıroğlu 3091. Fa

Arbutus unedo L., 12, 01.06.2011, M.Sağıroğlu 3093. Fa.

PRIMULACEAE

Primula acaulis (L.) L. subsp. acaulis, 29, 25.03.2012, M.Turna 1097. Hkrp. Avr.-Sib.

Primula acaulis (Sm.) Greuter \& Burdet subsp. rubra, 17, 25.03.2012, M.Turna 1105. Hkrp. Ök.

Cyclamen coum Miller subsp. coum, 29, 25.03.2012, M.Turna 1101. Hkrp.

Anagallis arvensis L. var. arvensis, 8, 05.05.2012, M.Turna 1153. Te.

Anagallis arvensis L. var. caerulea (L.) Gouan, 6, 25.05.2013, M.Turna 1483. Te.

EBENACEAE

Diospyros kaki L. fil., 7, 19.06.2011, M.Turna 1552. Fa. Kültür.

OLEACEAE

Ligustrum vulgare L., 12, 19.06.2011, M.Turna 1011. Fa. Avr.-Sib.

GENTIANACEAE

Blackstonia perfoliata (L.) Hudson subsp. serotina (W. Koch ex Reichb.) Vollmann, 6, 25.05.2013, M.Turna

1456. Hkrp.

Centaurium erythraea Rafn subsp. erythraea, 8, 22.07.2012, M.Turna 1296. Hkrp.

Centaurium erythraea Rafn subsp. turcicum (Valen.) Melderis, 8, 08.07.2013, M.Turna 1536. Hkrp. Gnş. Yay.

Gentiana asclepiadea L., 32, 20.09.2012, M.Turna 1342. Hkrp. Avr.-Sib.

CONVOLVULACEAE

Convolvulus cantabrica L., 6, 25.05.2013, M.Turna 1496. Hkrp.

Convolvulus arvensis L., 16, 31.07.2011, M.Turna 1071. Hkrp.

Convolvulus scammonia L., 8, 03.06.2012, M.Turna 1182. Hkrp. Akd.

Calystegia sepium (L.) R. Br. subsp. sepium, 32, 22.07.2012, M.Turna 1302. Hkrp.

Calystegia silvatica (Kit.) Griseb., 17, 03.06.2012, M.Turna 1222. Hkrp.

Ipomoea purpurea (L.) Roth, 12, 01.06.2011, M.Sağıroğlu3053. Hkrp.

BORAGINACEAE

Heliotropium dolosum De Not., 8, 20.06.2012, M.Turna 1258. Te.

Myosotis incrassata Guss., 12, 13.04.2013, M.Turna 1416. Te. Akd.

Myosotis arvensis (L.) Hill subsp. arvensis, 8, 05.05.2012, M.Turna 1165. Te. Avr.-Sib.

Myosotis sylvatica Ehrh. ex Hoffm. subsp. cyanea Vestergren, 43, 13.04.2013, M.Turna 1448. Hkrp.

Myosotis lithospermifolia Hornem., 31, 05.05.2012, M.Turna 1139. Hkrp.

Cynoglossum creticum Miller, 6, 25.05.2013, M.Turna 1467. Hkrp.

Echium italicum L., 8, 22.07.2012, M.Turna 1287. Hkrp. Akd. 
Echium vulgare L. subsp. vulgare, 23, 08.07.2013, M.Turna 1516. Hkrp. Avr.-Sib.

Echium angustifolium Miller, 31, 03.06.2012, M.Turna 1211. Hkrp. Akd.

Trachystemon orientalis (L.) G. Don, 29, 25.03.2012, M.Turna 1096. Hkrp. Ök.

SOLANACEAE

Solanum decipiens Opiz, 32, 20.09.2012, M.Turna 1330-1334. Te.

SCROPHULARIACEAE

Verbascum orientale (L.) All. subsp. orientale, 12, 19.06.2011, M.Turna 1006. Krp. Akd. Geniş yayılışl1.

Verbascum sinuatum L. var. sinuatum, 3, 08.07.2013, M.Turna 1503. Hkrp. Akd.

Verbascum bithynicum Boiss., 8, 08.07.2013, M.Turna 1517. Hkrp. Avr.-Sib. End.

Verbascum speciosum Schrader, 17, 19.06.2011, M.Turna 1045. Hkrp.

Scrophularia scopolii [Hoppe ex] Pers. var. scopolii, 16, 05.05.2012, M.Turna 1127; 8, 20.09.2012, M.Turna

1359; 34, 13.04.2013, M.Turna 1439. Hkrp. Gnş. Yay.

Linaria pelisseriana (L.) Miller, 12, 01.06.2011, M.Sağıroğlu 3058. Te. Akd.

Kickxia elatine (L.) Dumort. subsp. crinita (Mabille) Greuter, 6, 25.05.2013, M.Turna 1481. Te. Akd.

Veronica serpyllifolia L., 43, 13.04.2013, M.Turna 1424. Krp.

Veronica persica Poiret, 16, 25.03.2012, M.Turna 1108; 10, M.Turna 1389. Te.

Veronica anagallis-aquatica L. subsp. anagallis-aquatica, 43, 25.05.2013, M.Turna 1477. Hkrp. Genişyay1lışl1.

Veronica chamaedrys L., 31, 05.05.2012, M.Turna 1142. Hkrp. Avr.-Sib.

Odontites vulgaris Moench, 31, 20.09.2012, M.Turna 1344. Te. Avr.-Sib.

Bartsia trixago L., 6, 25.05.2013, M.Turna 1466. Te.

\section{OROBANCHACEAE}

Orobanche alba Stephan ex. Willd. subsp. alba, 29, 25.03.2012, M.Turna 1100. Vp.

VERBENACEAE

Verbena officinalis L. var. officinalis, 8, 20.06.2012, M.Turna 1260. Hkrp. Gnş. Yay.

\section{LABIATAE}

Teucrium chamaedrys L. subsp. chamaedrys, 8, 20.06.2012, M.Turna 1277a. Hkrp. Avr.-Sib.

Teucrium chamaedrys L. subsp. lydium O. Schwarz, 8, 20.06.2012, M.Turna 1277b. Hkrp. D.Akd.

Scutellaria altissima L. subsp. commutata (Guss.) Nyman, 12, 19.06.2011, M.Turna 1002. Hkrp. Avr.-Sib.

Scutellaria rubicunda Hornem. subsp. subvelutina (Rech. fil.) Edmondson, 12, 19.06.2011, M.Turna 1007.

Hkrp. D.Akd.

Phlomis russeliana (Sims) Lag. ex Benth., 12, 01.06.2011, M.Sağıroğlu 3102. Hkrp. Ök. End.

Lamium purpureum L. var. purpureum, 31, 05.05.2012, M.Turna 1116. Te. Avr.-Sib.

Galeobdolon luteum Hudson subsp. montanum (Pers.) R.Mill, 18, 05.05.2012, M.Turna 1146. Te. Avr.-Sib.

Stachys byzantina K.Koch, 6, 25.05. 2013, M.Turna 1465. Hkrp. Avr.-Sib.

Stachys maritima Gouan, 12, 01.06.2011, M.Sağıroğlu3047- 3101. Kam. Akd.

Stachys annua (L.) L. subsp. annua var. lycaonica Bhattacharjee, 34, 13.04.2013, M.Turna 1437. Hkrp. Ir.-Tur.

Stachys annua (L.) L. subsp. annua var. annua, 4, 25.05.2013, M.Turna 1473. Hkrp. Geniş yayılışli.

Prunella vulgaris L., 23, 19.06.2011, M.Turna 1044; 32, 22.07.2012, M.Turna 1305. Hkrp. Avr.-Sib. Geniş yayılışlı.

Prunella laciniata (L.) L., 12, 19.06.2011, M.Turna 1003 ; 15, 31.07.2011, M.Turna 1074. Hkrp. Avr.-Sib.

Origanum vulgare L. subsp. hirtum (Link) Ietswaart, 32, 20.09.2012, M.Turna 1345. Hkrp. Akd.

Calamintha sylvatica Bromf. subsp. ascendens (Jordan) P. W. Ball, 7, 22.07.2012, M.Turna 1279. Hkrp.

Calamintha nepeta (L.) Savi subsp. glandulosa (Req.) P. W. Ball, 32, 20.09.2012, M.Turna 1347; 2, M.Turna

1502. Hkrp..

Clinopodium vulgare L. subsp. vulgare, 15, 31.07.2011, M.Turna 1073. Hkrp.

Clinopodium vulgare L. subsp. arundanum (Boiss.) Nyman, 32, 22.07.2012, M.Turna 1304. Hkrp. Gnş. Yay.

Acinos rotundifolius Pers., 9, 01.06.2011, M.Sağıroğlu 3085. Te. Gnş. Yay.

Mentha pulegium L., 15, 31.07.2011, M.Turna 1078. Krp.

Mentha longifolia (L.) Hudson subsp. typhoides (Briq.) Harley var. typhoides, 31, 31.07.2011, M.Turna 1086.

Krp. Geniş yayılışli.

Mentha spicata L. subsp. spicata, 17, 18.07.2011, M.Turna 1066. Hkrp.

Mentha spicata (Briq.) Greuter \& Burdet subsp. condensata, 8, 20.09.2012, M.Turna 1353. Hkrp.

Ziziphora taurica Bieb. subsp. taurica, 16, 05.05.2012, M.Turna 1125. Te. Ir-Tur.

Salvia forskahlei L., 8, 22.07.2012, M.Turna 1281; 32, 20.09.2012, M.Turna 1335. Hkrp. Ök.

Salvia glutinosa L., 32, 17.10.2012, M.Turna 1374. Hkrp. Ök.

Salvia virgata Jacq., 12, 19.06.2011, M.Turna 1383. Hkrp. Ir.-Tur.

Salvia napifolia Jacq., 15, 31.07.2011, M.Turna 1070. Hkrp. Akd.

PLANTAGINACEAE

Plantago major L. subsp. major, 2, 08.07.2013, M.Turna 1518. Hkrp.

Plantago lanceolata L., 12, 19.06.2011, M.Turna, 1016. Hkrp. 


\section{THYMELAEACEAE}

Daphne pontica L.subsp. pontica, 15, 21.03.2013, M.Turna 1399. Kam. Ök.

LAURACEAE

Laurus nobilis L., 7, 13.04.2013, M.Turna 1449. Fa. Akd.

EUPHORBIACEAE

Euphorbia stricta L., 31, 22.07.2012, M.Turna 1312. Te. Avr.-Sib.

Euphorbia helioscopia L. subsp. helioscopia, 8, 05.05.2012, M.Turna 1178. Te.

Euphorbia cyparissias L., 8, 05.05.2012, M.Turna 1176. Hkrp. Avr.-Sib.

Euphorbia amygdaloides L. subsp. amygdaloides, 8, 05.05.2012, M.Turna 1177. Hkrp. Avr.-Sib.

ARISTOLOCHİACEAE

Aristolochia pontica Lam., 8, 21.03.2013, M.Turna 1388. Hkrp. Ök.

URTICACEAE

Urtica dioica L. subsp. dioica, 23, 19.06.2011, M.Turna 1057. Hkrp. Avr.-Sib.

MORACEAE

Morus alba L., 32, 08.07.2013, M.Turna 1525. Fa. Kültür.

Ficus carica L. subsp. carica, 17, 03.06.2012, M.Turna 1219. Fa. Gnş. Yay.

ULMACEAE

Ulmus glabra Hudson, 31, 19.06.2011, M.Turna 1384. Fa. Avr.-Sib.

UImus minor Miller subsp. minor, 16, 31.07.2011, M.Turna 1075. Fa.

JUGLANDACEAE

Juglans regia L., 17, 03.06.2012, M.Turna 1218. Fa.

PLATANACEAE

Platanus orientalis L., 8, 03.06.2012, M.Turna 1197. Fa. Gnş. Yay.

FAGACEAE

Fagus orientalis Lipsky, 43, 25.05.2013, M.Turna 1475. Fa. Avr.-Sib.

Castanea sativa Miller, 43, 25.05.2013, M.Turna 1474. Fa. Avr.-Sib.

Quercus robur L. subsp. robur, 12, 13.04.2013, M.Turna 1412. Fa. Avr.-Sib.

Quercus cerris L., 43, 13.04.2013, M.Turna 1404. Fa. Avr.-Sib.

CORYLACEAE

Carpinus betulus L., 32, 08.07.2013, M.Turna 1545. Fa. Avr.-Sib.

Carpinus orientalis Miller subsp. orientalis, 43, 13.04.2013, M.Turna 1433. Fa.

Corylus avellana L. var. pontica (C. Koch) Winkler, 16, 05.05.2012, M.Turna 1131. Fa. Ök.

BETULACEAE

Alnus glutinosa (L.) Gaertner subsp. glutinosa, 3, 08.07.2013, M.Turna 1506. Fa. Avr.-Sib.

SALICACEAE

Salix bornmuelleri Hausskn., 8, 21.03.2013, M.Turna 1392. Fa. Ir.-Tur.

Salix alba L. subsp. alba, 31, 03.06.2012, M.Turna 1201. Fa. Avr.-Sib. Gnş. Yay.

Salix caprea L., 32, 05.05.2012, M.Turna 1150. Fa. Avr.-Sib

Populus alba L. var. alba, 18, 05.05.2012, M.Turna 1149. Fa. Avr.-Sib

Populus tremula subsp. tremula L., 12, 13.04.2013, M.Turna 1415. Fa. Avr.-Sib

MONOCOTYLEDONAE

LILIACEAE

Fritillaria pontica Wahlenb., 41, 13.04.2013, M.Turna 1419. Krp. Avr.-Sib.

SMILACACEAE

Smilax excelsa L., 41, 13.04.2013, M.Turna 1430. Krp. Ök.

COLCHICACEAE

Colchicum speciosum Steven, 16, 25.03.2012, M.Turna 1112. Krp. Avr.-Sib.

ASPARAGACEAE

Ruscus hypoglossum L., 29, 25.03.2012, M.Turna 1099. Krp. Avr.-Sib.

Ruscus aculeatus L. 17, 05.05.2012, M.Turna 1180. Krp.

Asparagus acutifolius L., 8, 22.07.2012, M.Turna 1294. Krp. Akd.

Scilla autumnalis L., 44, 20.09.2012, M.Turna 1343. Krp. Akd.

Ornithogalum sigmoideum Freyn \& Sint., 8, 21.03.2012, M.Turna 1385. Krp. Avr.-Sib.

Ornithogalum orthophyllum subsp. kochii Ten, 16, 25.03.2012, M.Turna 1107. Krp. Gnş. Yay.

Muscari neglectum Guss. ex Ten, 16, 05.05.2012, M.Turna 1122. Krp. Gnş. Yay.

AMARYLLIDACEAE

Galanthus plicatus Bieb. subsp. byzantinus (Baker) D. A. Webb, 25, 25.03.2012, M.Turna 1114. Krp. Ök. End.

Allium paniculatum L. subsp. paniculatum, 6, 25.05.2013, M.Turna 1468. Krp. D. Akd.

Allium rupestre Steven, 6,M.Turna 1497. Avr.-Sib. Krp.

DIOSCOREACEAE 
Dioscorea communis (L.) Caddick \& Wilkin, 43, 13.04.2013, M.Turna 1413. Krp. JUNCACEAE

Juncus inflexus L., 24, 10.06.2011, M.Turna 1030; 27.11.2011, M.Turna 1090.

Hkrp. Gnş. Yay.

Juncus conglomeratus L., 4, 25.05.2013, M.Turna 1470. Krp. Avr.-Sib.

Luzula forsteri (Sm.) DC., 43, 13.04.2013, M.Turna 1411. Hkrp. Avr.-Sib.

CYPERACEAE

Isolepis setacea (L.) R. Br., 33, 17.10.2012, M.Turna 1375. Krp.

GRAMINEAE

Brachypodium pinnatum (L.) P. Beauv., 4, 25.05.2013, M.Turna 1548. Hkrp. Avr.-Sib.

Brachypodium distachyon (L.) P.Beauv, 4, 25.05.2013, M.Turna 1547. Te. Akd.

Elymus flaccidifolius (Boiss. \& Heldr.) Melderis, 31, 20.09.2012, M.Turna 1551. Hkrp. D.Akd.

Aegilops geniculata Roth, 4, 25.05.2013, M.Turna 1486. Te. Akd.

Hordeum murinum L. subsp. glaucum (Steudel) Tzvelev, 8, 03.06.2012, M.Turna 1236. Te

Bromus secalinus L., 32, 20.09.2012, M.Turna 1339. Te. Avr.-Sib.

Bromus racemosus L., 23, 19.06.2011, M.Turna 1051. Te. Avr.-Sib.

Bromus commutatus Schrader, 23, M.Turna 1049; 12, 19.06.2011, M.Turna 1014. Te.

Bromus hordeaceus L. subsp. hordeaceus, 12, 13.04.2013, M.Turna 1549. Te.

Bromus hordeaceus L. subsp. molliformis (Lloyd) Maire \& Weiller, 23, 19.06.2011, M.Turna 1053. Te. Akd.

Bromus hordeaceus L. subsp. thominii (Hardouin) Maire \& Weiller, 23, 19.06.2011, M.Turna 1050; 31,

20.09.2012, M.Turna 1328.Te. Akd.

Bromus arvensis L., 8, 22.07.2012, M.Turna 1293. Te.

Bromus sterilis L., 8, 05.05.2012, M.Turna 1174. Te. Gnş. Yay.

Bromus madritensis L., 32, 17.10.2012, M.Turna 1382. Te.

Bromus rubens L., 8, 20.09.2012, M.Turna 1363. Te.

Bromus diandrus Roth, 12, 01.06.2011, M.Sağıroğlu 3055. Te.

Avena barbata Pott ex Link subsp. barbata4, 25.05.2013, M.Turna 1487. Te. Akd.

Rostraria cristata (L.) Tzvelev var. cristata, 8, 03.06.2012, M.Turna 1235.

Rostraria cristata (L.) Tzvelev var. glabriflora ( Trautv.) Doğan, 8, 05.05.2012, M.Turna 1154. Hkrp.

Koeleria macrantha (Ledeb.) Schult, 43, 25.05.2013, M.Turna 1488. Hkrp. Gnş. Yay.

Holcus lanatus L., 8, 20.06.2012, M.Turna 1262. Hkrp. Avr.-Sib.

Apera spica-venti (L.) P. Beauv., 43, 25.05.2013, M.Turna 1484. Te. Avr.-Sib.

Agrostis castellana Boiss. \& Reuter subsp. byzantina (Boiss.) Hackel, 31, 27.11.2011, M.Turna 1087. Te.

Avr.Sib.

Polypogon viridis (Gouan) Breistr., 8, 20.09.2012, M.Turna 1362. Te. Avr.-Sib. Gnş. Yay.

Alopecurus myosuroides Huds. var. myosuroides, 6, 05.05.2012, M.Turna 1164 Gnş. Yay.

Phleum pratense L., 23, 27.11.2011, M.Turna 1088. Krp. Avr.-Sib.

Festuca valesiaca Schleich. ex Gaudin, 31, 03.06.2012, M.Turna 1243. Krp.

Lolium rigidum Gaudin var. rottbollioides Heldr. ex Boiss., 8, 03.06.2012, M.Turna 1232. Hkrp. Akd.

Vulpia myuros (L.) C. C. Gmel., 8, 05.05.2012, M.Turna 1239. Hkrp Gnş. Yay.

Catapodium rigidum (L.) C.E. Hubbard ex Dony subsp. rigidum var. majus (C.Presl) M.Laínz, 31, 13.04.2013, M.Turna 1550. Te.

Psilurus incurvus (Gouan) Schinz \& Thell., 8, 21.03.2013, M.Turna 1391. Te. Gnş. Yay.

Poa annua L., 42, 13.04.2013 M.Turna 1423.Te.

Poa trivialis L., 31, 03.06.2012, M.Turna 1244. Krp.

Poa pratensis L. 31, 03.06.2012, M.Turna 1233. Krp. Gnş. Yay.

Poa angustifolia L., 12, 19.06.2011, M.Turna 1009; 39, 03.06.2012, M.Turna 1241. Krp. Gnş. Yay.

Poa nemoralis L., 31, 03.06.2012, M.Turna 1242. Hkrp. Gnş. Yay.

Poa bulbosa L., 43, 13.04.2013, M.Turna 1406. Krp.

Dactylis glomerata L. subsp. glomerata, 23, 19.06.2011, M.Turna 1027. Krp. Avr.-Sib.

Cynosurus echinatus L., 12, 19.06.2011, M.Turna 1017. Krp. Akd.

Briza maxima L., 43, 25.05.2013, M.Turna 1546. Krp.

Sesleria alba Sm., 43, 13.04.2013, M.Turna 1405-1434. Krp.

Molinia caerulea (L.) Moench, 31, 20.09.2012, M.Turna 1338. Krp.

Stipa holosericea Trin., 4, M.Turna 1485. Krp. İr.-Tur.

Oryzopsis coerulescens (Desf.) Hack., 26, 08.07.2013, M.Turna 1520. Krp.

Phragmites australis (Cav. ) Trin. ex Steudel, 26, 08.07.2013, M.Turna 1533. Krp. Avr.-Sib.

Cynodon dactylon (L.) Pers. var. dactylon, 6, 08.07.2013, M.Turna 1537. Krp.

Tragus racemosus (L.) All., 8, 20.09.2012, M.Turna 1349. Te. 
Setaria viridis (L.) P.Beauv., 8, 20.09.2012, M.Turna 1350-1351. Te. Gnş. Yay.

Bothriochloa ischaemum (L.) Keng, 8, 20.09.2012, M.Turna 1352. Krp.

\section{Tartışma ve Sonuç}

Yapılan arazi çalışmaları ile toplam 608 bitki örneği toplanmıştır. Bu örneklerin teşhisi sonucunda 79 familyaya ait 257 cins ve bu cinslere ait 434 tür ve tür altı seviyede takson tespit edilmiştir. Tespit edilen 434 taksondan 292'si tür, 105'i alttür ve 37'si varyete seviyesindedir. Bu taksonlardan 9'u kültür, 5'i kozmopolit ve 62'si geniş yayılışlıdır.

Araştırma alanında tespit edilen 434 taksondan (tür ve tür altı) 5 tanesi Pteridophyta’ya dahil olup geriye kalan 429 tanesi Spermatophyta'ya aittir. Spermatophyta'ya ait olan bu taksonlardan 5 tanesi Gymnospermae, 424 tanesi ise Angiospermae' ye dahildir.

Angiospermae' den 357 takson Dicotylodonae, 67 takson ise Monocotyledonae aittir. Araştırma alanında takson sayısı bakımından en zengin familyalar Tablo 2 gösterilmiştir. Buna göre, İkramiye Vadisi (Sakarya) ve çevresinde 49 tür ve tür altı takson ile ilk sırayı Poaceae almaktadır. Daha sonra sırası ile Asteraceae (47 tür ve tür altı takson), Fabaceae (43 tür ve tür altı takson), Lamiaceae (28 tür ve tür altı takson), Brassicaceae (24 tür ve tür altı takson), Rosaceae (20 tür ve tür altı takson), Caryophyllaceae (15 tür ve tür altı takson) izlemektedir.

Tablo 2. Araştırma alanında tespit edilen takson sayısı bakımından en zengin familyalar.

\begin{tabular}{llll}
\hline Sira No & Familya Adı & Takson Sayısı & Oranı (\%) \\
\hline $\mathbf{1}$ & Poaceae & 49 & 11,29 \\
$\mathbf{2}$ & Asteraceae & 47 & 10,82 \\
$\mathbf{3}$ & Fabaceae & 43 & 9,90 \\
$\mathbf{4}$ & Lamiaceae & 28 & 6,45 \\
$\mathbf{5}$ & Brassicaceae & 24 & 5,52 \\
$\mathbf{6}$ & Rosaceae & 20 & 4,60 \\
$\mathbf{7}$ & Caryophyllaceae & 15 & 3,45 \\
\hline
\end{tabular}

En fazla cinse sahip olan familyalar; Poaceae 32 cins ile ilk sırada, Asteraceae 31 cins ile 2. sirada yer almaktadır. Daha sonra sirası ile Brassicaceae ve Fabaceae (17 cins), Rosaceae ve Lamiaceae (14 cins) ve Apiaceae (10 cins) izlemektedir.

Araştırma alanındaki tür ve tür altı taksonların fitocoğrafik bölgelere göre dağılımı Tablo 3' te verilmiştir. Araştırma alanında tespit edilen toplam 434 taksondan fitocoğrafik bölgesi belli olanların sayısı 175'dir. Fitocoğrafik bölgesi bilinenlerin 110'u Avrupa-Sibirya, 51'i Akdeniz, 14'ü İran-Turan elementidir. Öksin elementi olarak tespit edilen taksonlar Avrupa-Sibirya fitocorafik bölgesine dahil edilmiştir. Toplam 434 takson içerisinde Türkiye florasında fitocoğrafik bölgesi bilinmeyen takson sayısı 260’ dır.

Tablo 3: Araştırma alanında tespit edilen taksonların fitocoğrafik bölgelere göre dağılımı.

\begin{tabular}{lll}
\hline Fitocoğrafik Bölge & Takson Sayısı & Oranı (\%) \\
\hline Avrupa-Sibirya & 110 & 25,34 \\
Akdeniz & 51 & 11,75 \\
İran-Turan & 14 & 3,23 \\
Fitocoğrafik Bölgesi Bilinmeyenler & 259 & 59,68 \\
\hline
\end{tabular}

Araştırma alanında tespit edilen toplam 434 taksondan 46'sı Fanerofit, 32'si Kamefit, 173'ü Hemikriptofit, 58'i Kriptofit, 122'si Terofit ve 1 tanesi Vasküler parazittir. Araştırma alanında tespit edilen 434 taksondan 11' i endemik olup alandaki tür ve tür altı taksonların endemizm oranı \% 2,53'dür.

Tür ve tür altı seviyede en çok takson içeren cinsler incelenecek olursa, ilk sırayı 14 tür ve tür altı takson ile Trifolium ilk sırada yer almaktadır. Daha sonra sırasıyla Bromus (11 tür ve tür altı takson), Hypericum (8 tür ve tür altı takson), Geranium (7 tür ve tür altı takson), Lathyrus, Poa ve Galium (6 tür ve tür altı takson) izlemektedir. 
Araştırma sonucunda toplanan 434 takson, araştırma bölgesine yakın çevrelerde yapılan flora çalışmalarıyla karşılaştırılmıştır. Karşılaştırma yapılan çalışmalar Tablo 4’de verilmiştir.

Tablo 4: Araştırma alanı ile karşılaştırmayapılan çalışmalaf ve takson sayıları.

\begin{tabular}{lll}
\hline $\begin{array}{l}\text { Çalışma } \\
\text { No }\end{array}$ & Çalışma Adı (Yazarı ve yılı) & $\begin{array}{c}\text { Takson } \\
\text { Sayısı }\end{array}$ \\
\hline $\mathbf{1}$ & İkramiye Vadisi ve Çevresinin Florası & 434 \\
$\mathbf{2}$ & Kocaeli-Karamürsel-Yalakdere Beldesi ve Çevresinin Florası (Yılancı, 2013) & 409 \\
$\mathbf{3}$ & $\begin{array}{l}\text { Flora of Arslanbey (İzmit/Turkey) and Cultivated Plants in İzmit City Center } \\
\text { (Aslan ve Sağıroğlu, 2011) }\end{array}$ & 489 \\
$\mathbf{4}$ & $\begin{array}{l}\text { Vascular Plant Diversity in Geyve Gorge (Sakarya/Turkey) and its Environs } \\
\text { (Koyuncu vd., 2012) }\end{array}$ & 1150 \\
$\mathbf{5}$ & Beşkayalar Vadisi (Gölcük-Kocaeli)'nin Florası (Akaydın vd., 2006) & 293 \\
\hline
\end{tabular}

Karşılaştırma yapılan çalışmalarda takson sayısı bakımından ilk sırada 1150 takson ile Koyuncu ve arkadaşlarının (2012) çalışması, ikinci sırada 489 taksonla Aslan (2011)'nun çalışması, üçüncü sırada 434 taksonla bizim çalışmamız, dördüncü sırada 409 taksonla Yılancı (2013)'nın çalışması ve son sırada 293 taksonla Akaydın ve arkadaşlarının (2006) çalışması yer almaktadır.

Karşılaştııılan araştırma alanlarında yapılan çalışmalardaki takson sayılarında farklılıklar gözlenmektedir. Çalışma alanlarının büyüklüğü, çalışma alanının coğrafik durumu ve alan üzerindeki antropojenik etkiler bu farklılıkların ortaya çıkmasına neden olmaktadır.

Bizim çalışmamızda Poaceae içerdiği 49 takson ile ilk sırada yer almaktadır. Çalışma bölgesinde tespit edilen en büyük ilk 5 familya Poaceae, Asteraceae, Fabaceae, Lamiaceae ve Brassicaceae, Türkiye florasının da en büyük ilk 5 familyasını oluşturmaktadır. Araştırma alanında en fazla takson içeren cinsler ve yakın bölgelerde yapılan çalışmalar ile karşılaştırılması Tablo 5'de verilmiştir. Çalışmalar incelendiğinde, Trifolium (14 takson) cinsinin en fazla takson içererek ilk sırada yer aldığı görülmektedir. İkinci sırada Bromus (11 takson) ve üçüncü sırada Hypericum ( 8 takson) cinsi yer almaktadır. Trifolium Türkiye florasında en büyük cinsler arasında 9. sırada yer almaktadır. Türkiye florasında en büyük cinslerden Astragalus ve Verbascum araştırma alanında en büyük cinsler arasında tespit edilememiştir. Türkiye florasında en büyük cinsler arasında olan Galium çalışmamızda da en büyük cinsler içinde yer almaktadır. Bu farklılıklar araştırma alanının daha küçük, daha homojen bir yapıya sahip olmasından kaynaklanmaktadır.

Tablo 5. Araştırma alanında en fazla takson içeren cinsler ve yakın bölgelerde yapılan çalışmalar ile karşılaştırılması.

\begin{tabular}{|c|c|c|c|c|c|c|c|c|c|c|}
\hline \multirow[t]{3}{*}{ Cins } & \multicolumn{10}{|c|}{ Araştırmalar } \\
\hline & \multicolumn{2}{|c|}{1} & \multicolumn{2}{|l|}{2} & \multicolumn{2}{|l|}{3} & \multicolumn{2}{|l|}{4} & \multicolumn{2}{|l|}{5} \\
\hline & 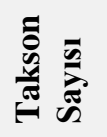 & ప & 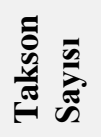 & పే & 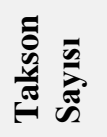 & పే & 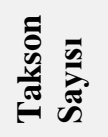 & 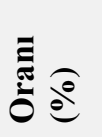 & 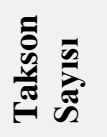 & పే \\
\hline Trifolium & 14 & 3,22 & 6 & 1,46 & 11 & 2,24 & 15 & 1,30 & 5 & 1,70 \\
\hline Bromus & 11 & 2,53 & 2 & 0,48 & 5 & 1,02 & 4 & 0,34 & - & - \\
\hline Hypericum & 8 & 1,84 & 5 & 1,22 & 3 & 0,61 & 10 & 0,87 & 5 & 1,70 \\
\hline Geranium & 7 & 1,61 & 6 & 1,46 & 2 & 0,40 & 8 & 0,70 & 3 & 1,02 \\
\hline Lathyrus & 6 & 1,38 & 5 & 1,22 & 2 & 0,40 & 11 & 0,96 & 2 & 0,68 \\
\hline Poa & 6 & 1,38 & 1 & 0,24 & 2 & 0,40 & 6 & 0,52 & 3 & 1,02 \\
\hline Galium & 6 & 1,38 & 4 & 0,97 & 1 & 0,20 & 7 & 0,60 & 2 & 0,68 \\
\hline
\end{tabular}

Çalışmalarda en fazla cins içeren familyalar incelendiğinde ilk sırayı Asteraceae almaktadır. 3, 4 ve 5. çalışmalarda Poaceae, 2. çalışmada ise Fabaceae 2. Sirada yer almaktadır. En fazla cins içeren familyalar incelenen çalışmalarda farklılık göstermektedir. Bunu nedeni, çalışılan bölgelerin farklı iklim ve vejetasyon özelliklerine sahip olmasıdır. 
Araştırma alanında tespit edilen taksonların fitocoğrafik bölgelere göre dağılımı karşılaştırıldığında, çalışmaların hepsinde ilk sırada Avrupa-Sibirya fitocoğrafik bölgesi, 2. Sırada Akdeniz fitocoğrafik bölgesi ve 3. sırada IranTuran fitocoğrafik bölgesi yer almaktadır (Tablo 6). 2,3,4, ve 5. çalışmaların yapıldığı alanlar ve araştırma alanımız Avrupa-Sibirya floristik bölgesi içinde yer almaktadır, bu yüzden bu bölgelerde yayılış gösteren bitkilerinin çoğunun Avrupa-Sibirya elementi olduğu gözlenmiştir.

Tablo 6: Araştırma alanında tespit edilen taksonların fitocoğrafik bölgelere göre dağılımı ve yakın bölgelerde yapılan çalışmalarla karşılaştırılması.

\begin{tabular}{|c|c|c|c|c|c|c|c|c|c|c|}
\hline \multirow{3}{*}{$\begin{array}{l}\text { Fitocoğrafik } \\
\text { Bölge }\end{array}$} & \multicolumn{10}{|c|}{ Araştırmalar } \\
\hline & \multicolumn{2}{|c|}{1} & \multicolumn{2}{|l|}{2} & \multicolumn{2}{|l|}{3} & \multicolumn{2}{|l|}{4} & \multicolumn{2}{|l|}{5} \\
\hline & 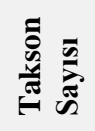 & పే & 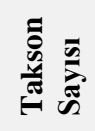 & పే & 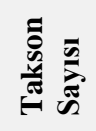 & పే & 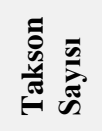 & పేస & 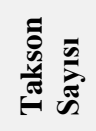 & ప્డ \\
\hline $\begin{array}{l}\text { Avrupa- } \\
\text { Sibirya }\end{array}$ & 110 & 25.34 & 87 & 21.02 & 102 & 20.85 & 148 & 15.88 & 85 & 29 \\
\hline Akdeniz & 51 & 11.75 & 64 & 15.89 & 60 & 12.26 & 107 & 11.48 & 30 & 9.8 \\
\hline Iran-Turan & 14 & 3.23 & 7 & 1.71 & 11 & 2.24 & 57 & 6.12 & 3 & 1 \\
\hline $\begin{array}{l}\text { Fitocoğrafik } \\
\text { Bölgesi } \\
\text { Bilinmeyen }\end{array}$ & 259 & 59.68 & 251 & 61.36 & 317 & 64.82 & 620 & 66.52 & 169 & 58 \\
\hline
\end{tabular}

Tablo 7. Araştırma alanında tespit edilen endemik takson sayısı ve endemizm oranının yakın bölgelerde yapılan çalışmalar ile karşılaştırılması.

\begin{tabular}{llllll}
\hline & Araştırmalar & & & \\
\cline { 2 - 6 } & $\mathbf{1}$ & $\mathbf{2}$ & $\mathbf{3}$ & $\mathbf{4}$ & $\mathbf{5}$ \\
\hline $\begin{array}{l}\text { Endemik } \\
\begin{array}{l}\text { Takson } \\
\text { Sayısı }\end{array}\end{array}$ & 11 & 8 & 11 & 72 & 6 \\
$\begin{array}{l}\text { Endemizm } \\
\text { Oranı (\%) }\end{array}$ & 2.53 & 1.95 & 2.55 & 6.26 & 2 \\
\hline
\end{tabular}

Tablo 8. Endemik taksonlar ve tehlike kategorileri.

\begin{tabular}{ll}
\hline Takson & $\begin{array}{l}\text { Tehlike } \\
\text { Kategorisi }\end{array}$ \\
\hline Abies nordmanniana (Stev.) Spach subsp. bornmuelleriana (Mattf.) Coode \& Cullen & LC \\
Thlaspi jaubertii Hedge & NE \\
Lathyrus undulatus Boiss. & VU \\
Asperula pestalozzae Boiss. & LC \\
Campanula lyrata Lam. subsp. lyrata & LC \\
Campanula ajugifolia Schult. & NE \\
Campanula latiloba A. DC. & LC \\
Campanula grandis Fisch. \& C.A.Mey. subsp. grandis & LC \\
Verbascum bithynicum Boiss. & NE \\
Phlomis russeliana (Sims) Bentham & LC \\
Galanthus plicatus Bieb. subsp. byzantinus (Baker) D. A. Webb & NE \\
\hline
\end{tabular}

Araştırma alanında tespit edilen endemik takson sayısı ve endemizm oranı yakın bölgelerde yapılan çalışmalar ile karşılaştırıldığında (Tablo 7); 2, 3 ve 5. çalışmaların endemizm oranları ile benzerlik gösterdiği görülmüştür. 4. çalışma ile diğer çalışmaların endemizm oranları arasında \% 3.5-4.5 'luk fark bulunmaktadır. Çalışma alanındaki endemizm oranı ülkemizin endemizm oranından $(\% 31,5)$ oldukça düşüktür. 
Türkiye florasında endemik bitkilerin fitocoğrafik bölgelere dağılımına bakıldığında Iran-Turan fitocoğrafik bölgesi ilk sırada gelir. Bunu Akdeniz ve Avrupa-Sibirya fitocoğrafik bölgeleri takip eder. Çalışma alanının Avrupa-Sibirya fitocoğrafik bölgesi içerisinde bulunması endemizm oranının düşük olmasının ilk sebebidir. Diğer sebepler araştırma bölgesinde yerleşim yerinin çok olması, tarım alanlarının bulunması nedeniyle geniş yayılışlı taksonların çok bulunması olabilir.

Sonuç olarak çalışma alanında ve benzer alanlarda gözlenen aşırı otlatma, tarım alanı oluşturma, yerleşim alanı oluşturma, ormanların yakılması ve ağaçların kesilmesi, doğal yayılış gösteren bitkilerin bilinçsizce toplanması doğada tahribata neden olmaktadır ve biyoçeşitliliğe zarar vermektedir.

\section{Kaynaklar}

1. Acartürk, R.,(2001). Süs Bitkileri ve Yer Örtücüleri, Ankara, .

2. Akayadın, G., Çalıșkan, G., Yılmaz, E. B.,(2006). Beşkayalar Vadisi (Gölcük/Kocaeli )'nin Florası, Fırat Üniv. Fen ve Müh. Bil. Dergisi, 18(4), 459-469.

3. Akman, Y., (2011). İklim ve Biyoiklim, Palme Yayınları, Ankara.

4. Aslan, O., Sağıroğlu, M., (2011).Flora of Arslanbey (İzmit, Turkey) and Cultivated Plants in İzmit City Center, Biological Diversity and Conservation, 4(2), 172-184.

5. Baytop, A.,(1998). İngilizce-Türkçe Botanik Kılavuzu, İ.Ü. Basımevi ve Film Merkezi, İstanbul, 1-375.

6. Brummitt, R.K., Powell, C.E., (1999) Authors of Plant Names, The Royal Botanic Gardens, Kew, USA, 1732,

7. Davis, P.H., (1965-1985) Flora of Turkey and the East Aegean Islands, Edinburgh Univ. Press., Edinburgh, Vol. 1-9.

8. Davis, P.H., Mill, R.R., Tan, K.,(1988) Flora of Turkey and the East Aegean Islands, Edinburgh Univ. Press., Edinburgh, Vol. 10.

9. Davis, P.H. ve Hedge, I.C., (1975). The Flora of Turkey; Past, Present and Future, Candollea, 30, 331-351.

10. Güner, A., Aslan, S., Ekim, T., Vural, M., Babaç, M. T. (edtr.) (2012). Türkiye Bitkileri Listesi (Damarlı Bitkiler). Nezahat Gökyiğit Botanik Bahçesi ve Flora Araştırma Derneği Yayını, İstanbul.

11. Koyuncu, O., Yaylacı, Ö.K., Öztürk, D., Tokur, S., (2012) Vascular Plant Diversity in Geyve Gorge (Sakarya/Turkey) and its Environs, Biological Diversity and Conservation, 5, 3,

12. Namıkoğlu, N. G., (2010). Türkiye’nin Ağaçları ve Çalıları, NTV Yayınları, İstanbul,

13. Sağıroğlu, M., Olgaç, E., Ertürk, B. ve Turna, M.. (2012). An ethnobotanical survey from Şile (İstanbul) and Karasu (Sakarya),OT Sistematik Botanik Dergisi, 19(2). 93-104.

14. Sağıroğlu, M., Köseoğlu, Toksoy S. ve Turna, M. (2017). Medicinal Plants in Flora of Ikramiye Valley (Sapanca/Sakarya/Turkey). Sakarya University Journal of Science, 21(3), 527-539.

15. Yılancı, M.S., Sağıroğlu, M.,(2013). Kocaeli-Karamürsel-Yalakdere Beldesi ve Çevresinin Florası, SAU Fen Bil. Der., 17(3), 405-423.

16. Stearn, W.T., (1973) Botanical Latin: History, Grammar Syntax, Terminology and Vocabulary, Great Britain by Redwood Burn Limited Trowbridge \& Esher, 379.

17. www.ipni.org, 10.04.2020.Available: http://www.earth.google.com. [Erişildi: 804 2020]. 